\title{
Development of a multichannel far-infrared tangential interferometer/ polarimeter for the National Spherical Torus Experiment
}

\author{
B. H. Deng, H. C. Chen, C. W. Domier, ${ }^{a)}$ M. Johnson, K. C. Lee, N. C. Luhmann, Jr., \\ and B. R. Nathan \\ Department of Applied Science, University of California at Davis, 3001 Engineering Unit III, Davis, \\ California 95616 \\ H. Park \\ Princeton Plasma Physics Laboratory, Princeton, New Jersey 08543
}

(Presented on 8 July 2002)

Completion of a multichannel far-infrared (FIR) tangential interferometer/polarimeter is under way for the National Spherical Torus Experiment, for the measurement of plasma electron density and toroidal magnetic-field distributions. The system is based on the three-wavelength configuration, which was first developed by Rommers and Howard in the RTP tokamak [Plasma Phys. Controlled Fusion 38, 1805 (1996)]. The probe beams enter the plasma through the windows on the midplane in Bay-K, and are reflected back to the detectors by retro-reflectors. The three FIR beams with a wavelength of $119 \mu \mathrm{m}$ are produced by three Methanol lasers optically pumped by a $\mathrm{CO}_{2}$ laser tuned to $9.7 \mu \mathrm{m}$. The entire system is designed to have a full remote control capability using the LABVIEW program and fiber links. The technical details of the system development will be presented together with preliminary experimental result. (c) 2003 American Institute of Physics.

[DOI: $10.1063 / 1.1538359]$

\section{INTRODUCTION}

Interferometry and polarimetry are widely utilized as standard diagnostics for high-temperature plasmas. ${ }^{1}$ It was proposed by Park et al. ${ }^{2}$ that a far-infrared (FIR) interferometer/polarimeter is an ideal tool for simultaneous measurement of electron density and toroidal magnetic-field distribution in the National Spherical Torus Experiment (NSTX). Numerical studies presented in Ref. 2 show that the optimum probe beam wavelength is $119 \mu \mathrm{m}$, at which the plasma will give high signal-to-noise ratio signals for detection, while the refraction effects are negligible. For this system, two channels have been operational since mid 2000, one additional channel was added in June 2001, and the remaining channels are being installed and will be operational before December 2002. Here, the technical details of the system, including the characteristics of the lasers, the optical design, the remote control system, and the plan for the final stage of system implementation will be described in Sec. II, sample data of fast magnetohydrodynamics signals from the existing channels which are free from machine vibration will be presented in Sec. III, followed by a brief summary.

\section{DEVELOPMENT OF INTERFEROMETER/ POLARIMETER SYSTEM}

\section{A. Laser system}

A triple FIR laser configuration ${ }^{1}$ is adopted for simultaneous measurement of plasma-induced phase changes (density measurement) and polarization changes (Faraday rotation, toroidal magnetic-field measurement), as shown in Fig.

${ }^{a)}$ Electronic mail: cwdomier@ucdavis.edu
1. This configuration has the advantage of high-temporal resolution for Faraday measurements. ${ }^{1}$ The polarization of one of the two laser beams is rotated by $90^{\circ}$ with a half wave plate before the two beams are combined with a polarizer. The combined laser beams are then turned into left- and right-hand circularly polarized beams for probing the plasma. Since the two beams have slightly detuned frequencies, their phase shifts due to the plasma can be determined independently. The average of the phase shifts will be the interferometer phase shift, while the difference will be twice the Faraday rotation angle. ${ }^{1}$ A third linearly polarized laser beam is utilized as the local oscillator.

Using the predicted plasma parameters of NSTX, the optimum probe beam wavelength was found to be $119 \mu \mathrm{m}$. The interferometer phase shift was expected to be less than 30 fringes, the Faraday rotation angle to be less than $40^{\circ}$, and the refraction of the beam less than $0.5 \mathrm{~mm} .^{2}$ The probing laser beams are produced by two optically pumped $\mathrm{CH}_{3} \mathrm{OH}$ lasers from the MIRI system for TFTR in PPPL. ${ }^{3}$ The cavities of these lasers consist of a copper mirror with a $6 \mathrm{~mm}$ diameter hole in the center for the input of $\mathrm{CO}_{2}$ pump beam, and a giant hole output coupler ${ }^{4}$ with a coupling hole of 11 $\mathrm{mm}$ in diameter. To be able to design the optics with confidence, the propagation characteristics of the laser beams in the far field are measured in detail. Shown in Fig. 2 are the beam patterns of FIR\#1 laser measured at different distances from the laser output coupler. It is seen that the laser beam propagates essentially as a Gaussian beam in the far field. The local oscillator beam is produced by a Stark-tuned methanol laser, also from PPPL. ${ }^{5}$ The application of the Stark-tuned laser allowed us to design a system at IF fre- 


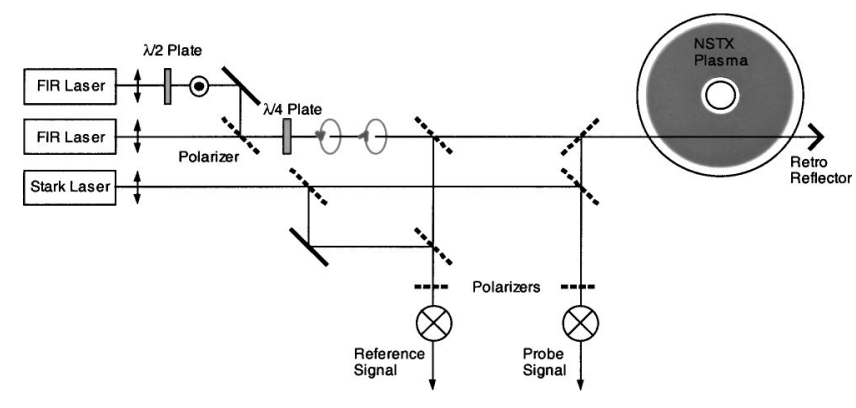

FIG. 1. Schematic showing the triple FIR laser configuration.

quencies of 3, 4, and $7 \mathrm{MHz}$. Details of this application will be discussed separately. ${ }^{6}$

A high-power $\mathrm{CO}_{2}$ laser provided by UC Davis is utilized as the pump source of the FIR lasers. It is grating tuned to the desired 9P36 line $(\lambda=9.695 \mu \mathrm{m})$. It has a unique fourelectrode design, which is safe to operate, and does not require Brewster windows. ${ }^{7}$ For the previous two- and threechannel operation, the laser output power was around $55 \mathrm{~W}$ continuous wave (CW) due to aging. Recently, this has been replaced by a newly refurbished similar laser producing stable power above $90 \mathrm{~W}$. This gives a maximum FIR power of $60 \mathrm{~mW}$ for the probe beam, and $32 \mathrm{~mW}$ for the LO beam, which will be sufficient for the envisioned full seven-channel operation. Fine tuning of the lasers are still going on and can be further improved.

The entire system is designed to have a full remote control capability. The FIR laser cavities are controlled by stepper motors, either manually by toggle switches in the test cell, or remotely by a fiber link connecting to the serial port of the $\mathrm{PC}$ in the control room. The $\mathrm{CO}_{2}$ laser cavity can also be locally or remotely controlled by adjusting the bias voltage to the piezoelectric transducer (PZT) unit in the laser head. The bias voltage is provided by the Stanford Research System's dc power supply, which has a GPIB interface, and a remote analog control input. The GPIB equipment are connected to the PC in the control room via National Instruments' GPIB-Ethernet converter, and fiber Ethernet link. The system diagnostic signals are wired to plugins of the Agilent HP3499A switch/control unit located in the NSTX test cell, and can be monitored by using the LABVIEW program in the

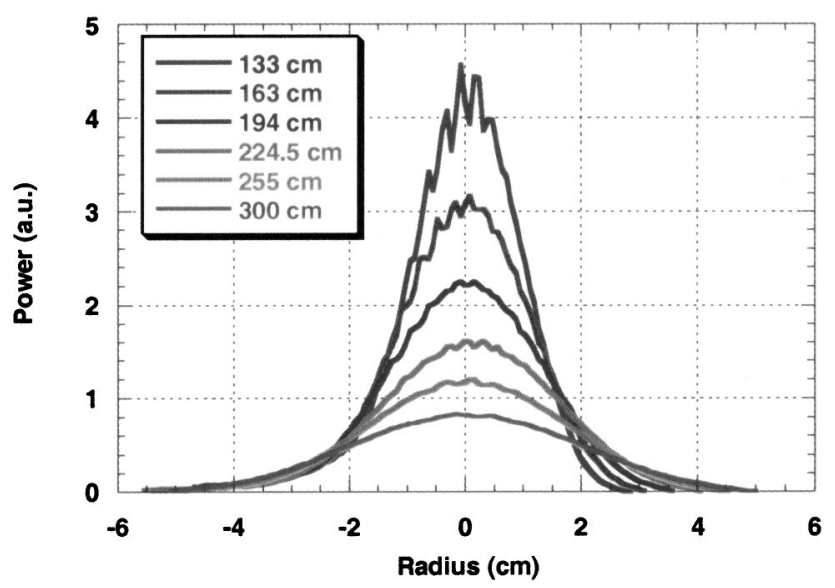

FIG. 2. Beam propagation patterns of the FIR\#1 laser output in the far field.

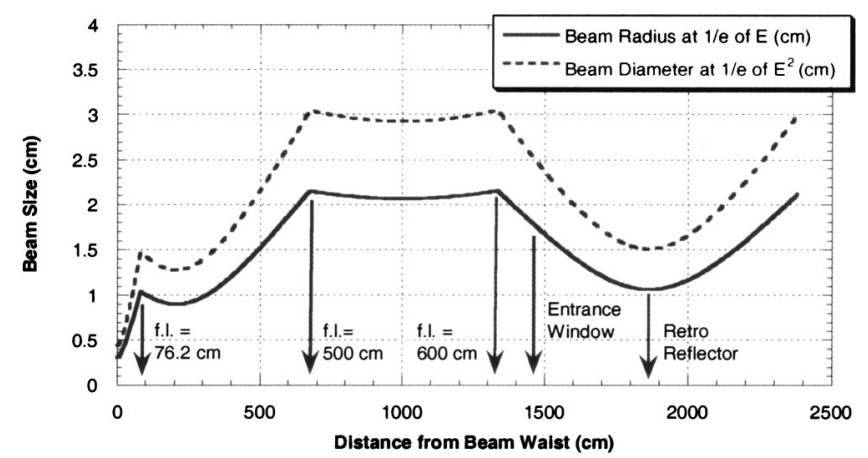

FIG. 3. FIR laser beam propagation, collimation, and focusing diagram. The beams are collimated by the Gaussian beam telescope consisted of $f_{1}(f . l$. $=76.2 \mathrm{~cm})$ and $f_{2}(f . l .=500 \mathrm{~cm})$ lens.

control room via GPIB commands. The HP3499A is also utilized to remotely turn on/off the $\mathrm{CO}_{2}$ laser power supply and control the gas pressure. For safety considerations, two local and one remote emergency stop buttons are installed in the interlock loop for the $\mathrm{CO}_{2}$ laser power supply. In addition, a video camera is installed to visually monitor the system performance.

\section{B. Optical system and mechanical frame}

From the measured propagation characteristics of the FIR laser beams, a Gaussian beam telescope consisting of two focusing mirrors $\left(f_{1}\right.$ and $\left.f_{2}\right)$ are designed to collimate the beam, and a third focusing mirror $\left(f_{3}\right)$ is utilized to focus the beam onto the retro-reflector, as shown in Fig. 3. The beam splitting and detection optics of all seven channels are arranged between $f_{1}$ and $f_{3}$. The collimated beam size is chosen so that a significant fraction of the optics from the TFTR MIRI system ${ }^{3}$ can be reused. The focal length of the third focusing mirror is different for each channel, to accommodate the different chord lengths, and to simplify the mirror arrangements.

The overall structure of the system is shown in Fig. 4. It

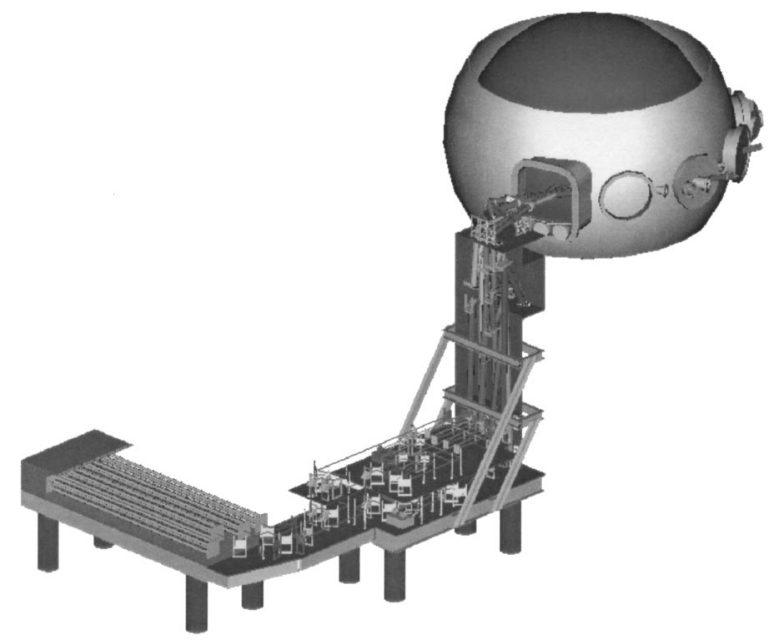

FIG. 4. Perspective view of the FIReTIP system (to scale). The NSTX machine structure is simplified, but with accurate relative positions. The three channels have been operating have tangency of 32,57 , and $150 \mathrm{~cm}$, respectively. The remaining channels have tangency of $85,103,123$, and $137 \mathrm{~cm}$, respectively. 


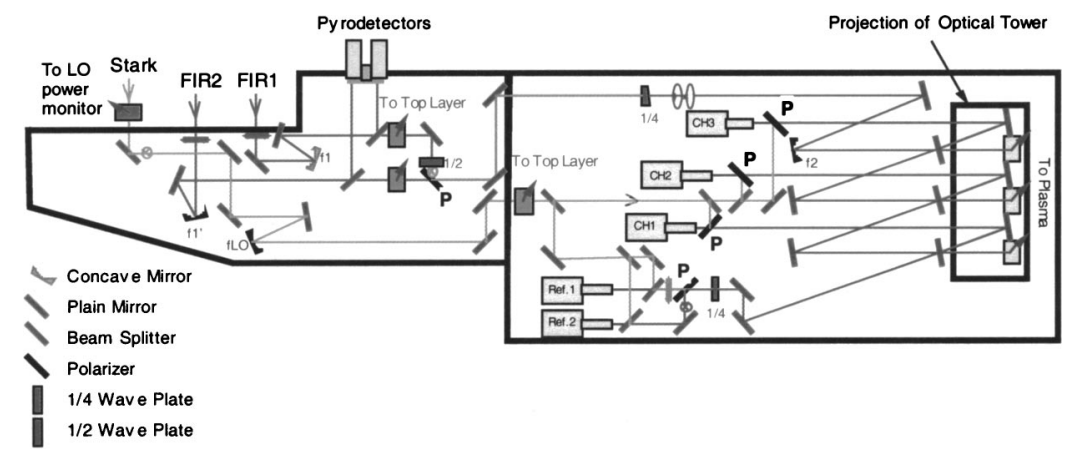

FIG. 5. Optical layout on the bottom layer of the optical table, which supports the first three channels and reference channels.

is designed with AUTOCAD. The details of the system, including the lasers, optical tables, optical tower, optical mounts, the Bay- $\mathrm{K}$ window and flange, and the relative positions are all drawn to scale. The $\mathrm{CO}_{2}$ optics, i.e., the lens, mirrors, and beam splitters at the far end of the laser table are enclosed by an aluminum box lined with graphite plates, so that the system can be treated as embedded for safety. A temperature sensor is installed inside the enclosure to detect the failure of the optics such as the falling of a mirror, and is wired to the laser power supply interlock loop. The optics for FIR beam splitting, combining, collimation, and signal detection mixers are arranged on a horizontal table, with the first layer installed for the first three channels and reference detectors. The second (top) layer optics will soon be installed for the remaining channels. The third focusing mirror for each channel and turning mirrors interfacing the retro reflectors and the optical table are arranged within a vertical tower structure made of G-10. The probe beams enter the plasma via 3 in. diameter quartz windows on the Bay-K flange, reach out to the retro reflectors via $1.5 \mathrm{in}$. diameter exit windows, and return along the same path to the optical table for detection.

The optical arrangement on the bottom layer of the optical table is shown in Fig. 5. It is seen that three probe signal mixers and two reference mixers are affixed to this layer. It is important that, after the probe beams are converted into circularly polarized beams, the optical components not introduce polarization changes. This is achieved by using the mesh beam splitters at a small incidence angle $\left(10^{\circ}\right),{ }^{8}$ as shown in Fig. 5 (the beam splitters in front of the optical tower). The optics and mixers of other channels will be installed on a second layer above this existing layer, with a similar configuration. In order to minimize the FIR propaga-

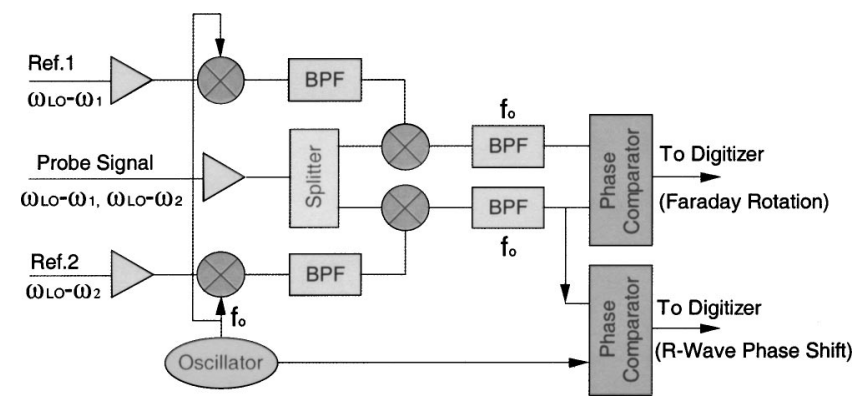

FIG. 6. Block diagram of the phase detection circuit. Using the tracking receiver technique, the frequency drift of the IF signals can be compensated. tion loss, all of the FIR optics and beam path are enclosed by acrylic plates, with dry nitrogen filling to control the humidity.

\section{Signal processing and data acquisition}

The tracking receiver technique is utilized in the phase detection circuit of far-infrared tangential interferometer/ polarimeter (FIReTIP), as shown in Fig. 6. Using the mixers and narrow-band bandpass filters, the IF signals carrying the plasma-induced phase information are all converted to a highly stable crystal oscillator frequency $f_{0}$, while preserving the phase. Therefore, the frequency jitter of the FIR lasers are compensated. The phase comparators have a linear output up to 64 fringes, and a frequency response of $<100$ kHz.

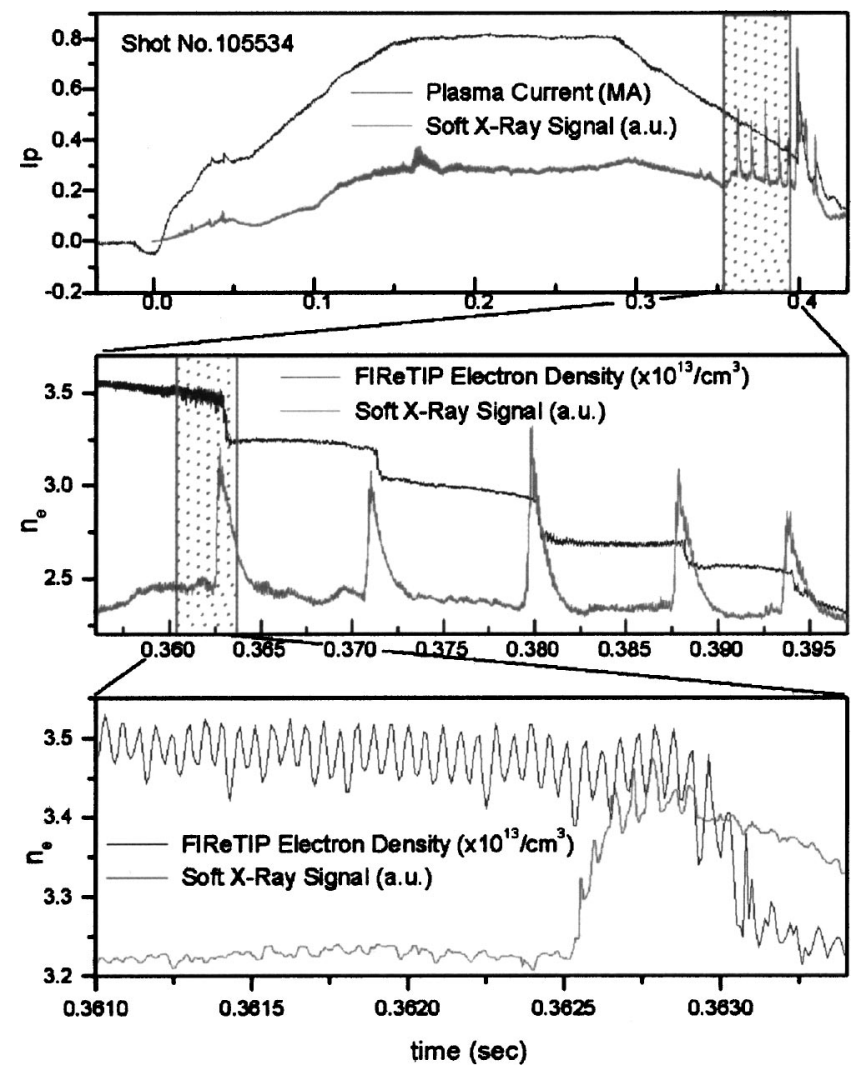

FIG. 7. Measurement of sawteeth and Mirnov oscillations by the FIReTIP system on NSTX. 

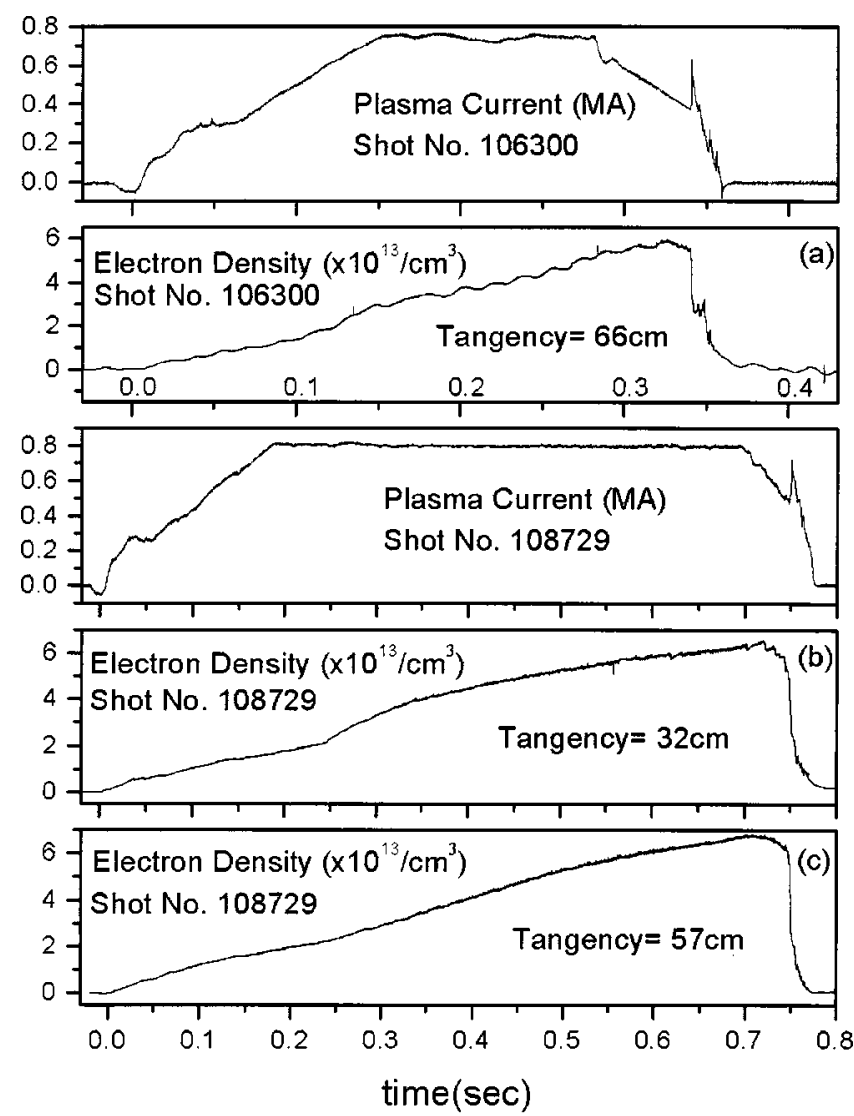

FIG. 8. Time traces of FIReTIP density measurements before (a) and after $[(\mathrm{b})$ and (c)] the installation of vibration free stands.

The outputs of the phase comparators are fed into standard DSP CAMAC digitizers (TRAQ 2812), and the data of each discharge are stored in standard DSP CAMAC memory modules (TRAQ 5000/5003A), and then transferred to the main computer of NSTX data acquisition system.

\section{INITIAL EXPERIMENTAL RESULT AND SUMMARY}

Two channels of the FIReTIP system have been operational since mid 2000, while one additional channel was added in June 2001. This partial system has been operating successfully. An example is shown in Fig. 7, where the sawteeth and Mirnov oscillations are clearly observed by the system.

In the early stage of the operation, sometimes lowfrequency oscillations were seen in the FIReTIP signals, as shown in Fig. 8(a). Therefore, the lasers, optical table, and tower are later supported by air pressured legs, and the retroreflectors are mounted on specially designed anti-vibration devices. These led to vibration free measurements of the system, as can been seen from Figs. 8(b) and 8(c). More data will be analyzed and will be presented separately. ${ }^{6}$

In summary, a multichannel, far-infrared, tangential interferometer/polarimeter system for NSTX is under development. It is aimed at plasma density and toroidal magneticfield measurements. $\mathrm{A} \mathrm{CO}_{2}$ laser is utilized to optically pump three far-infrared lasers with a wavelength of $119 \mu \mathrm{m}$. The triple-laser configuration is adopted for high-temporal resolution measurement of Faraday rotation. The system can be remotely operated by fiber links between the PC in the control room, and the lasers and equipments in the test cell. Three channels have been operational and good data are obtained. In the final stage of the system development, a highpower $\mathrm{CO}_{2}$ laser has recently been installed, and the FIR laser power improved. It will be sufficient for the full channel operation, which will be completed before December 2002.

\section{ACKNOWLEDGMENTS}

This work is supported by U.S. Department of Energy Grant No. DOE DE-FG03-99ER54518. Support from the NSTX team of PPPL is acknowledged.

${ }^{1}$ J. H. Rommers and J. Howard, Plasma Phys. Controlled Fusion 38, 1805 (1996).

${ }^{2}$ H. Park, C. W. Domier, W. R. Geck, and N. C. Luhmann, Jr., Rev. Sci. Instrum. 70, 710 (1999)

${ }^{3}$ D. K. Mansfield et al., Appl. Opt. 26, 4469 (1987).

${ }^{4}$ D. T. Hodges, F. B. Foote, and R. D. Reel, Appl. Phys. Lett. 29, 662 (1976).

${ }^{5}$ D. K. Mansfield et al., Appl. Phys. Lett. 31, 5030 (1992).

${ }^{6} \mathrm{~K}$. C. Lee, Rev. Sci. Instrum. (these proceedings).

${ }^{7}$ T. M. Lehecka, M.S. thesis, University of California, Los Angeles, California, 1986.

${ }^{8}$ Y. S. Hwang and H. K. Park, Appl. Opt. 28, 4999 (1989). 